\title{
AN ELEMENTARY INTEGRAL SOLUTION OPERATOR FOR THE CAUCHY-RIEMANN EQUATIONS ON PSEUDOCONVEX DOMAINS IN $\mathrm{C}^{n}$
}

\author{
BY \\ R. MICHAEL RANGE ${ }^{1}$
}

\begin{abstract}
An integral representation formula for $(0, q)$ forms is constructed on a strictly pseudoconvex domain $D$ in $\mathbf{C}^{n}$ by using only the local geometry of the boundary of $D$. By combining this representation with elementary results about compact operators in Banach spaces, one obtains the solution of the Levi problem and, more importantly, an integral solution operator for $\bar{\partial}$ on $D$. The construction does not need any a priori knowledge of the solvability of $\bar{\partial}$ and thus allows us to establish fundamental global results by a direct and elementary method.
\end{abstract}

1. Introduction. The classical methods for solving the Cauchy-Riemann equations on pseudoconvex domains in $\mathbf{C}^{n}$ use either the solution of the Levi problem and the Oka-Cartan theory of holomorphically convex domains (typically including a fair amount of sheaf theory) or abstract methods from the theory of partial differential equations. By using the solution of $\bar{\partial}$, it has then been possible to construct integral solution operators (analogous to the Cauchy transform in $\mathbf{C}^{1}$ ) on strictly pseudoconvex domains. These operators yield more refined estimates for solutions than those available through the classical methods.

The question arises whether one could develop integral solution operators and their numerous applications directly without making use of the classical methods for solving $\bar{\partial}$. In this paper we answer this question affirmatively by presenting an elementary construction of a solution operator for $\bar{\partial}$ on a strictly pseudoconvex domain $D$ in $\mathbf{C}^{n}$.

The construction involves two steps. First, one introduces an explicit integral operator $E_{q}$ which extends $\bar{\partial}$-cohomology classes of type $(0, q), q \geqslant 1$, from $D$ to a larger domain. The operator $E_{q}$ is obtained by generalizing the reproducing kernel for holomorphic functions given by Kerzman and Stein [6] to the case of $(0, q)$-forms. Combining the extension operator $E_{q}$ with basic properties of compact operators in Banach spaces, one immediately obtains a simple solution of the Levi problem. ${ }^{2}$ The

Received by the editors December 29, 1981.

1980 Mathematics Subject Classification. Primary 32A25, 32F15, 32F20; Secondary 35C15, 35N15.

Key words and phrases. Strictly pseudoconvex domains, integral representations, Levi problem, Hefer's lemma, integral solution operator for $\bar{\partial}$.

'Author partially supported by NSF grant MCS 81-02216.

${ }^{2}$ The author discovered this construction of the extension operator $E_{q}$ shortly after the work of Kerzman and Stein. In the meantime, Harvey and Polking [3] obtained the solution of the Levi problem by a similar construction, and by using information about Cauchy principal values of certain singular integrals, together with the finiteness theorem of Schwartz for Fréchét spaces.

(C)1982 American Mathematical Society 0002-9947/81/0000-0271/\$02.75 
principle of this proof is really the same as Grauert's [2], however the details are quite different.

Via the extension operator $E_{q}$, one has thus reduced the problem of solving $\bar{\partial} u=f$ on $D$ to the case where $f$ is $\bar{\partial}$-closed on a strictly pseudoconvex neighborhood $D_{\delta}$ of $\bar{D}$. In the second step one solves this latter problem. Briefly, the idea is as follows. It is well known how to construct an integral solution operator in the case of convex domains. So, in the spirit of Oka [9], we embed a suitable neighborhood $V$ of $\bar{D}$ into a convex domain $U$ in a higher-dimensional $\mathbf{C}^{N}$, and pull back the canonical global support function for $U$ to $V$. The main difficulty then is to obtain the required factorization (i.e. Hefer's lemma). It turns out that finiteness of $\bar{\partial}$-cohomology is sufficient for that.

By a result of Fornaess [1], one can even embed the domain $D$ itself into a convex domain in some $\mathbf{C}^{m}$; however, the proof, as well as the applications to integral kernels in [1], uses the solution of $\bar{\partial}$. In comparison, the embedding result used here is almost trivial, once one has solved the Levi problem.

This paper deals only with strictly pseudoconvex domains in $\mathbf{C}^{n}$. But once one has solved $\bar{\partial}$ on such domains, one can easily prove the Runge property for sublevel sets of a strictly plurisubharmonic exhaustion function, following the classical arguments of Oka for polynomially convex sets. In this way one can then solve the $\bar{\partial}$-equation on arbitrary pseudoconvex domains in $\mathbf{C}^{n}$. Furthermore, these methods can be extended to complex manifolds, leading to the characterization of Stein manifolds in terms of strictly plurisubharmonic exhaustion functions. This requires, essentially, only the finiteness of the $\bar{\partial}$-cohomology. To show that the $\bar{\partial}$-cohomology is actually 0 in this case gets somewhat more complicated, due to the lack of a simple generalization of the Bochner-Martinelli-Koppelman formula to Stein manifolds.

In conclusion, I would like to thank Ingo Lieb for several stimulating discussions regarding the problem dealt with in this paper.

2. The extension of $\bar{\partial}$-cohomology classes. Let $D$ be a strictly pseudoconvex domain in $\mathbf{C}^{n}$ with boundary $b D$ of class $C^{3}$, and let $r$ be a $C^{3}$ defining function for $D$, defined on a neighborhood of $\bar{D}$, which is strictly plurisubharmonic in a neighborhood of $b D$. Define

$$
F(z, \zeta)=2 \sum_{j=1}^{n} \frac{\partial r}{\partial \zeta_{j}}(\zeta)\left(\zeta_{j}-z_{j}\right)-\sum_{j, k=1}^{n} \frac{\partial^{2} r}{\partial \zeta_{j} \partial \zeta_{k}}(\zeta)\left(\zeta_{j}-z_{j}\right)\left(\zeta_{k}-z_{k}\right)
$$

The following result is a well-known consequence of Taylor's theorem and the strict plurisubharmonicity of $r$.

2.1 LEMMA. There are positive constants $\varepsilon$ and $c$, such that

$$
\operatorname{Re} F(z, \zeta) \geqslant-r(z)+c|z-\zeta|^{2}
$$

for all $(z, \zeta)$ with $\zeta \in b D$ and $|z-\zeta| \leqslant \varepsilon$.

Choose $\chi \in C^{\infty}\left(\mathbf{C}^{n} \times \mathbf{C}^{n}\right)$ such that $0 \leqslant \chi \leqslant 1, \chi(z, \zeta)=1$ for $|z-\zeta| \leqslant \varepsilon / 2$ and $\chi(z, \zeta)=0$ for $|z-\zeta| \geqslant \varepsilon$. For $j=1,2, \ldots, n$, define

$$
P_{j}(z, \zeta)=\chi\left[2 \frac{\partial r}{\partial \zeta_{j}}(\zeta)-\sum_{k=1}^{n} \frac{\partial^{2} r}{\partial \zeta_{j} \partial \zeta_{k}}(\zeta)\left(\zeta_{k}-z_{k}\right)\right]+(1-\chi)\left(\bar{\zeta}_{j}-\bar{z}_{j}\right)
$$


Then

$$
\phi(z, \zeta)=\sum_{j=1}^{n} P_{j}(z, \zeta)\left(\zeta_{j}-z_{j}\right)=\chi \cdot F+(1-\chi)|z-\zeta|^{2}
$$

has the following properties for any fixed $\zeta \in b D$ :

$$
\operatorname{Re} \phi(z, \zeta) \geqslant-r(z)+c|z-\zeta|^{2} \text { for }|z-\zeta| \leqslant \varepsilon / 2
$$

there is $\delta>0$ (independent of $\zeta$ ) such that $|\phi(z, \zeta)| \geqslant \delta$ for all $z$ with $r(z) \leqslant \delta$ and $|z-\zeta| \geqslant \varepsilon / 2$;

(2.4) $\phi(\cdot, \zeta)$ and $P_{j}(\cdot, \zeta), 1 \leqslant j \leqslant n$, are holomorphic on $\{z:|z-\zeta|<\varepsilon / 2\}$.

For $\lambda \in I=[0,1]$ and $\zeta \in b D$, we define

$$
w_{j}(z, \zeta, \lambda)=\lambda \frac{P_{j}(z, \zeta)}{\phi(z, \zeta)}+(1-\lambda) \frac{\bar{\zeta}_{j}-\bar{z}_{j}}{|\zeta-z|^{2}}, \quad 1 \leqslant j \leqslant n .
$$

By (2.3), $w_{j}$ has no singularities for $z \in D \cup\{z: r(z) \leqslant \delta,|z-\zeta| \geqslant \varepsilon / 2\}$. Furthermore

$$
\sum_{j=1}^{n} w_{j} \cdot\left(\zeta_{j}-z_{j}\right)=1
$$

Finally, set $W=\sum_{j=1}^{n} w_{j} d \zeta_{j}$.

For $q=0,1, \ldots, n-1$, the Cauchy-Fantappié form $\Omega_{q}(W)$ associated to the $(1,0)$ form $W$ is defined by

$$
\Omega_{q}(W)=\frac{(-1)^{q(q-1) / 2}}{(2 \pi i)^{n}}\left(\begin{array}{c}
n-1 \\
q
\end{array}\right) W \wedge\left(\underset{n-q-1}{\wedge} \bar{\partial}_{\zeta, \lambda} W\right) \wedge\left(\wedge_{q}^{\wedge} \bar{\partial}_{z} W\right) .
$$

We also set $\Omega_{-1}=\Omega_{n}=0 . \Omega_{q}(W)$ is a double differential form in $(\zeta, \lambda) \in b D \times I$ and $z \in D$ of degree $2 n-q-1$ in $(\zeta, \lambda)$ and type $(0, q)$ in $z$ (so differentials in $z$ commute with differentials in $(\zeta, \lambda)$ ). For $\lambda=0, \Omega_{q}(W)$ agrees with the BochnerMartinelli-Koppelman kernel [7] of type $(0, q)$ which is defined on $\mathbf{C}^{n} \backslash\{z=\zeta\}$. The following results are well known (see, for example, [10] and the references given there).

2.6 LEMMA. $\bar{\partial}_{\zeta, \lambda} \Omega_{q}(W)=(-1)^{q} \bar{\partial}_{z} \Omega_{q-1}(W)$ for any $W=\sum_{j=1}^{n} w_{j} d \zeta_{j}$ which satisfies (2.5).

2.7 Proposition. Let $0 \leqslant q \leqslant n, \Omega_{q}=\Omega_{q}(W)$, as defined above, and $f \in C_{0, q}^{1}(\bar{D})$. Then, for $z \in D$,

$$
f(z)=\int_{b D \times\{1\}} f(\zeta) \wedge \Omega_{q}(\zeta, z)+\bar{\partial}_{z} T_{q} f(z)+T_{q+1} \bar{\partial} f(z),
$$

where

$$
T_{q} f=\int_{b D \times I} f \wedge \Omega_{q-1}-\int_{D \times\{0\}} f \wedge \Omega_{q-1} .
$$

Let $\mu(\zeta)=(\zeta, 1)$. For $f \in C_{0, q}^{0}(b D)$, we define $E_{q} f=\int_{b D} f \wedge \mu^{*} \Omega_{q}(W)$. 
2.8 Lemma. For $q \geqslant 1$ and $f \in C_{0, q}^{0}(b D), E_{q} f$ is defined and of class $C^{\infty}$ on $\overline{D_{\delta}}=\{z: r(z) \leqslant \delta\}$. Moreover, for each multi-index $\alpha$, there is a constant $c_{\alpha}<\infty$, such that

$$
\operatorname{Sup}_{D_{\delta}}\left|D^{\alpha} E_{q} f(z)\right| \leqslant c_{\alpha}|f|_{L^{\infty}(b D)} \quad \text { for all } f \in C_{0, q}^{0}(b D) .
$$

Proof. By (2.4), the components of $W(\zeta, z, 1)$ are holomorphic on $\{z:|z-\zeta|<$ $\varepsilon / 2\}$. Hence, for $z \in \bar{D}_{\delta}$ fixed $\mu^{*} \Omega_{q}(W)=0$ for $|\zeta-z|<\varepsilon / 2$, and, by (2.3), any derivative with respect to $z$ of $\mu^{*} \Omega_{q}(W)$ is uniformly bounded for $z \in \bar{D}_{\delta}$ and $\zeta \in b D$ with $|\zeta-z| \geqslant \varepsilon / 2$.

2.9 LEMMA. If $f \in C_{0, q}^{1}(\bar{D}), q \geqslant 1$, and $\bar{\partial} f=0$ on $D$, then $\bar{\partial}_{z}\left(E_{q} f\right)(z)=0$ on $D_{\delta}$.

Proof. For $z \in D$, the statement follows directly from (2.7); by continuity it thus holds also for $z \in \bar{D}$. Fix $z_{0} \in D_{\delta} \backslash \bar{D}$. Since $\Omega_{q}$ and $\Omega_{q+1}$ are smooth for $\zeta \in b D$ and $z$ in the neighborhood of $z_{0}$, by (2.6) and Stokes' theorem, one obtains

$$
\bar{\partial}_{z}\left(E_{q} f\right)=(-1)^{q+1} \int_{b D} f \wedge \bar{\partial}_{\zeta}\left(\mu^{*} \Omega_{q+1}\right)=\int_{b D} \bar{\partial} f \wedge \mu^{*} \Omega_{q+1}=0
$$

in a neighborhood of $z_{0}$. Q.E.D.

Combining (2.7) and (2.9) one sees that $E_{q} f$ defines an extension of the $\bar{\partial}$ cohomology class in $H^{q}(D, \mathcal{O})$ of $f \in C_{0, q}^{1}(\bar{D})$ with $\bar{\partial} f=0$ to $H^{q}\left(D_{\delta}, \mathcal{\theta}\right)$. We will need the following information about regularity properties of $T_{q}$.

2.10 Proposition. For any $k=0,1,2, \ldots$ and $q \geqslant 1$, if $f \in C_{0, q}^{0}(\bar{D}) \cap C_{0, q}^{k}(D)$, one has $T_{q} f \in C_{0, q-1}^{k}(D)$. Moreover, there is a constant $C<\infty$, independent of $f$, such that

$$
\operatorname{Sup}_{D}\left|T_{q} f(z)\right| \leqslant C|f|_{L^{\infty}(\bar{D})} .
$$

Proof. Since $\phi(z, \zeta)=F(z, \zeta)$ for $|z-\zeta| \leqslant \varepsilon / 2$, one can estimate the kernels, as in the classical case, using (2.2) (Henkin [5], for $n=2$ and $q=1$; Lieb [8] for the general case).

2.11 REMARK. $T_{q}$ is even smoothing of order $\frac{1}{2}$ in the following sense:

$$
\left|T_{q} f\right|_{\operatorname{Lip}_{1 / 2}(D)} \leqslant C|f|_{L^{\infty}(\bar{D})} \text {. }
$$

For a proof see the estimations in [10].

3. The Levi problem. We consider the vectorspace

$$
Z_{q}^{1}(\bar{D})=\left\{f \in C_{0, q}^{1}(\bar{D}), \bar{\partial} f=0\right\}
$$

and the $C^{1}$ norm on $Z_{q}^{1}(\bar{D})$ given by

$$
\left\|\sum f_{J} d \bar{z}^{J}\right\|_{1}=\sum_{J} \sum_{|\alpha| \leqslant 1} \sup _{\bar{D}}\left|D^{\alpha} f_{J}(z)\right| .
$$

$Z_{q}^{1}$ with this norm is a Banach space. By Lemmas 2.8 and 2.9 we obtain

3.1 LEMMA. For $q \geqslant 1$ the restriction of $E_{q}$ to $Z_{q}^{1}(\bar{D})$ defines a compact linear operator $E_{q}: Z_{q}^{1} \rightarrow Z_{q}^{1}$. 
3.2 THEOREM. For $q \geqslant 1$ the operator $L_{q}=\bar{\partial} T_{q}$ defines a bounded linear transformation $L_{q}: Z_{q}^{1}(\bar{D}) \rightarrow Z_{q}^{1}(\bar{D})$ whose range has finite codimension.

Proof. By Proposition 2.7, $L_{q}=I-E_{q}$ on $Z_{q}^{1}$, where $I=$ identity. Since $E_{q}$ is compact, the theorem follows from standard facts about compact operators in Banach spaces.

3.3 ThEOREM. Let $D \subset \subset \mathbf{C}^{n}$ be strictly pseudoconvex with $C^{3}$-boundary. Then $D$ is holomorphically convex.

Proof. Fix $\zeta \in b D$. By (2.2)-(2.4), $h(z)=\phi(z, \zeta) \in C^{\infty}(\bar{D}), h(\zeta)=0, h(z) \neq 0$ for $z \in \bar{D} \backslash\{\zeta\}$, and $h$ is holomorphic for $|z-\zeta|<\varepsilon / 2$. Therefore, $f_{j}=\bar{\partial}\left(1 / h^{j}\right) \in$ $Z_{1}^{1}(\bar{D})$ for $j=1,2, \ldots$ By Theorem 3.2 there exist constants $c_{1}, \ldots, c_{l} \in \mathbf{C}, c_{l} \neq 0$, and a $(0,1)$ form $g \in Z_{1}^{\mathrm{l}}(\bar{D})$ such that $\sum_{j=1}^{l} c_{j} f_{j}=L_{1} g=\overline{\mathrm{\partial}} T_{1} g$. By $(2.10), T_{1} g \in$ $C^{1}(D)$ and $\sup _{D}\left|T_{1} g(z)\right|<\infty$. Therefore $H=\sum_{j=1}^{l} c_{j} / h^{j}-T_{1} g$ is holomorphic on $D$ and $\lim _{z \rightarrow \zeta}|H(z)|=\infty$. Q.E.D.

4. Factorization. A compact set $K \subset \mathrm{C}^{n}$ is called pseudoconvex if $K$ is the intersection of open pseudoconvex sets in $\mathbf{C}^{n}$. Equivalently, $K$ is pseudoconvex if and only if $K$ has a neighborhood basis of strictly pseudoconvex open sets with $C^{\infty}$ boundary. Note that the closure of a strictly pseudoconvex open set is pseudoconvex.

4.1 Proposition. Let $K \subset \mathbf{C}^{n}$ be a compact pseudoconvex set. Let $K_{1}=K \cap\{z$ : $\left.z_{1}=0\right\}$. Then every $f \in \mathcal{O}\left(K_{1}\right)$ has an extension $E f \in \mathcal{O}(K)$ such that $E f=f$ in $a$ neighborhood of $K_{1}$ in $\left\{z \in \mathbf{C}^{n}: z_{1}=0\right\}$.

Proof. Choose a neighborhood $W$ of $K$ such that $f$ is holomorphic on $W_{1}=W \cap$ $\left\{z: z_{1}=0\right\}$. Let $\pi: \mathbf{C}^{n} \rightarrow \mathbf{C}^{n}$ be defined by $\pi(z)=\left(0, z_{2}, \ldots, z_{n}\right)$. Since $W_{1}$ and $W \backslash \pi^{-1}\left(W_{1}\right)$ are disjoint and closed (relative to $W$ ), there is $\chi \in C^{\infty}(W)$ such that $\chi \equiv 1$ in a neighborhood of $W_{1}$ and $\chi \equiv 0$ in a neighborhood of $W-\pi^{-1}\left(W_{1}\right)$. For $j=1,2, \ldots, \alpha_{j}=\bar{\partial} \chi(f \circ \pi) / z_{1}^{j}$ is a well-defined, $\bar{\partial}$-closed $C_{0,1}^{\infty}$ form on $W$. Choose $D$ strictly pseudoconvex with smooth boundary such that $K \subset D \subset \subset W$. Then $\alpha_{j} \in$ $Z_{1}^{1}(\bar{D})$ for $j=1,2, \ldots$ and, by Theorem 3.2 , there are $C_{1}, \ldots, C_{l} \in \mathbf{C}, C_{l} \neq 0$, and $g \in Z_{1}^{1}(\bar{D})$ such that $\Sigma_{j=1}^{l} C_{j} \alpha_{j}=\bar{\partial} T_{1} g$. Dividing by a nonzero constant, we may assume $C_{l}=1$. Thus

$$
\bar{\partial}\left(z_{1}^{l} T_{1} g\right)=\sum_{j=1}^{l} C_{j} z_{1}^{l-j} \bar{\partial} \chi(f \circ \pi)
$$

and, hence, $E f=\sum_{j=1}^{l} C_{j} z_{1}^{l-j} \chi f \circ \pi-z_{1}^{l} T_{1} g$ is holomorphic on $D$ and satisfies $E f\left(0, z_{2}, \ldots, z_{n}\right)=f\left(0, z_{2}, \ldots, z_{n}\right)$. Q.E.D.

One can now repeat, with minor modifications, the arguments of Hefer [4] to prove the following result.

4.2 TheOREM. Let $K$ be a pseudoconvex compact set in $\mathbf{C}^{n}$. Given $f \in \mathcal{O}(K)$, there are $Q_{j} \in \mathcal{O}(K \times K), 1 \leqslant j \leqslant n$, such that

$$
f(w)-f(z)=\sum_{j=1}^{n} Q_{j}(z, w)\left(w_{j}-z_{j}\right) \quad \text { for }(z, w) \in K \times K .
$$


5. The solution of $\bar{\partial}$ on strictly pseudoconvex domains. We first solve the $\bar{\partial}$-equation on holomorphically convex compact subsets of a strictly pseudoconvex domain.

5.1 TheOREM. Let $D \subset \subset \mathbf{C}^{n}$ be strictly pseudoconvex and let $K \subset D$ be compact with $\hat{K}_{\mathcal{O}(D)}=K$. There are a neighborhood $V$ of $K$ and linear integral operators $R_{q}$ : $C_{0, q}^{0}(D) \rightarrow C_{0, q-1}^{0}(V), q=1,2, \ldots, n$, such that:

(i) for any $k=0,1,2, \ldots$, if $f \in C_{0, q}^{k}(D)$, the $R_{q} f \in C_{0, q-1}^{k}(V)$;

(ii) if $f \in C_{0, q}^{1}(D)$ and $\bar{\partial} f=0$, then $\bar{\partial}\left(R_{q} f\right)=f$ on $V$.

Proof. Let $\omega \subset \subset D$ be a strictly pseudoconvex neighborhood of $K$. There are finitely many functions $h_{1}, \ldots, h_{N} \in \mathcal{O}(D)$ such that the analytic polyhedron $A=\{z$ $\left.\in \omega:\left|h_{k}(z)\right|<1,1 \leqslant k \leqslant N\right\}$ satisfies $K \subset A \subset \subset \omega$. Let $\Delta^{N}$ be the open unit polydisc in $\mathbf{C}^{N}$. The map $H=\left(h_{1}, \ldots, h_{N}\right): A \rightarrow \Delta^{N}$ is proper and, by adding additional functions to the $h_{j}$ 's if necessary, one can also assume that $H$ is regular and one-to-one. Since $\Delta^{N}$ is convex, there is a convex neighborhood $U \subset \subset \Delta^{N}$ of $H(K)$ which can be taken to have smooth boundary transversal to the submanifold $H(A)$ of $\Delta^{N}$. Let $\rho \in C^{\infty}\left(\Delta^{N}\right)$ be a defining function for $U$. For $t \in \mathbf{C}^{N}$ and $\eta \in \Delta^{N}$, define

$$
G(t, \eta)=\sum_{k=1}^{N} \frac{\partial \rho}{\partial \eta_{k}}(\eta)\left(\eta_{k}-t_{k}\right)
$$

Because of the convexity of $U, G(t, \eta) \neq 0$ for $(t, \eta) \in U \times b U . V=H^{-1}(U) \subset \subset A$ is a neighborhood of $K$ with smooth boundary, and $\phi(z, \zeta)=G(H(z), H(\zeta)) \neq 0$ for $(z, \zeta) \in V \times b V$. Since $h_{k} \in \mathcal{O}(\bar{\omega})$, Theorem 4.2 gives functions $Q_{j k} \in \vartheta(\bar{\omega} \times \bar{\omega})$, $1 \leqslant j \leqslant n, 1 \leqslant k \leqslant N$, such that

$$
h_{k}(\zeta)-h_{k}(z)=\sum_{j=1}^{n} Q_{j k}(z, \zeta)\left(\zeta_{j}-z_{j}\right) ;
$$

therefore, the functions

$$
P_{j}(z, \zeta)=\sum_{k=1}^{N} \frac{\partial \rho}{\partial \eta_{k}}(H(\zeta)) Q_{j k}(z, \zeta)
$$

are holomorphic in $z \in \omega$, and

$$
\sum_{j=1}^{n} P_{j}(z, \zeta)\left(\zeta_{j}-z_{j}\right)=\phi(z, \zeta)
$$

Now set

$$
w_{j}(z, \zeta, \lambda)=\lambda \frac{P_{j}(z, \zeta)}{\phi(z, \zeta)}+(1-\lambda) \frac{\bar{\zeta}_{j}-\bar{z}_{j}}{|\zeta-z|^{2}}
$$

on $V \times b V \times[0,1]$, and $W=\sum_{j=1}^{n} w_{j} d \zeta_{j}$. Finally, define the kernels $\Omega_{q}(W), q \geqslant 0$, as in $\S 2$. Observe that for $q \geqslant 1, \Omega_{q}(W)=0$ for $(\zeta, \lambda) \in b V \times\{1\}$, as $W$ is holomorphic in $z$ on $V$ for such $(\zeta, \lambda)$. Therefore, by Proposition 2.7, the operator

$$
R_{q} f=\int_{b V \times I} f \wedge \Omega_{q-1}(W)-\int_{V \times\{0\}} f \wedge \Omega_{q-1}(W)
$$


solves the $\bar{\partial}$-equation on $V$ and hence on $K \subset V$. This proves (ii) of the theorem; (i) follows from 2.10 or directly from the above representation of $R_{q} f$.

5.3 REMARK. It is immediate from (5.2) and the definition of $\Omega_{q-1}(W)$ that $R_{q}$ has the following smoothing property:

$$
\left|R_{q} f\right|_{\operatorname{Lip}_{\alpha}(K)} \leqslant C_{\alpha}|f|_{L^{\infty}(\bar{V})} \quad \text { for any } \alpha<1 .
$$

We now combine Theorem 5.1 with the extension operator $E_{q}$ of $\S 2$. Let $D \subset \subset \mathbf{C}^{n}$ be strictly pseudoconvex with $C^{3}$-boundary. For $q \geqslant 1$ construct $E_{q}: C_{0, q}^{0}(b D) \rightarrow$ $C_{0, q}\left(\bar{D}_{\delta}\right)$ and $T_{q}: C_{0, q}^{0}(\bar{D}) \rightarrow C_{0, q-1}^{0}(D)$ as in $\S 2$. Note that $D_{\delta}$ can be chosen strictly pseudoconvex, and hence holomorphically convex, by Theorem 3.3. Let $K=\hat{\bar{D}}_{\mathcal{O}\left(D_{\delta}\right)} \cdot{ }^{3}$ Now apply Theorem 5.1 to $K$ and $D_{\delta}$, obtaining the operator $R_{q}: C_{0, q}^{0}\left(D_{\delta}\right) \rightarrow$ $C_{0, q-1}^{0}(K)$. Set $T_{q}+R_{q} E_{q}=S_{q}$; then $S_{q}: C_{0, q}^{0}(\bar{D}) \rightarrow C_{0, q-1}^{0}(D)$. The main properties of $S_{q}$ are summarized in the following theorem.

5.4 TheOREM. (i) For any $k=0,1,2, \ldots, S_{q} f \in C_{0, q-1}^{k}(D)$ for $f \in C_{0, q}^{0}(\bar{D}) \cap$ $C_{0, q}^{k}(D)$.

(ii) If $f \in C_{0, q}^{0}(\bar{D}) \cap C_{0, q}^{1}(D)$ and $\bar{\partial} f=0$, then $\bar{\partial}\left(S_{q} f\right)=$ fon $D$.

(iii) $\left|S_{q} f\right|_{\operatorname{Lip}_{1 / 2}(D)} \leqslant C|f|_{L^{\infty}(\bar{D})}$ for $f \in C_{0, q}^{0}(\bar{D})$.

Proof. (i) follows from 2.10, 2.8 and 5.1(i); (ii) follows from 2.7, 2.9 and 5.1(ii); finally, (iii) follows from 2.8, 2.11 and 5.3.

5.5 COROllaRY. Let $K \subset \mathbf{C}^{n}$ be a pseudoconvex compact set ${ }^{4}$ and $q \geqslant 1$. Then for every $\bar{\partial}$-closed $C_{0, q}^{\infty}$ form $f$ on a neighborhood of $K$ there is a $C_{0, q-1}^{\infty}$ form $u$ such that $\bar{\partial} u=f$ on a neighborhood of $K$.

In terms of sheaf cohomology this can be reformulated as follows.

5.6 Corollary. Let $K \subset \mathbf{C}^{n}$ be a pseudoconvex compact set. Then $H^{q}(K, \theta)=0$ for $q \geqslant 1$.

\section{REFERENCES}

1. J. E. Fornaess, Embedding strictly pseudoconvex domains in convex domains, Amer. J. Math. 98 (1976), 529-569.

2. H. Grauert, On Levi's problem and the embedding of real-analytic manifolds, Ann. of Math. (2) 68 (1958), 460-472.

3. R. Harvey and J. Polking, Fundamental solutions in complex analysis. I: The Cauchy-Riemann operator, Duke Math. J. 46 (1979), 253-300.

4. H. Hefer, Zur Funktionentheorie mehrerer Veränderlichen. Über eine Zerlegung analytischer Funktionen und die Weil'sche Integraldarstellung, Math. Ann. 122 (1950), 276-278.

5. G. M. Henkin, Integral representation of functions in strictly pseudoconvex domains and applications to the $\bar{\partial}$-problem, Math. USSR-Sb. 11 (1970), 273-281.

6. N. Kerzman and E. M. Stein, The Szegö kernel in terms of Cauchy-Fantappié kernels, Duke Math. J. 45 (1978), 197-224.

\footnotetext{
${ }^{3}$ In fact $\hat{\bar{D}}_{\mathcal{O}_{\left(D_{\delta}\right)}}=\bar{D}$, but this is not needed here.

${ }^{4}$ See the beginning of $\$ 4$.
} 
7. W. Koppelman, The Cauchy integral for differential forms, Bull. Amer. Math. Soc. 73 (1967), 554-556.

8. I. Lieb, Die Cauchy-Riemannschen Differentialgleichungen auf streng pseudokonvexen Gebieten, Math. Ann. 190 (1970), 6-44.

9. K. Oka, Sur les fonctions analytiques de plusieurs variables. I: Domaines convexes par rapport aux fonctions rationelles, J. Sci. Hiroshima Univ. Ser. A 6 (1936), 245-255.

10. R. M. Range and Y. T. Siu, Uniform estimates for the $\bar{\partial}$-equation on domains with piecewise smooth strictly pseudoconvex boundaries, Math. Ann. 206 (1973), 325-354.

Department of Mathematics, State University of New York at Albany, Albany, New York 12222 